\title{
Phosphatidylinositide 3-Kinase Contributes to the Anti-Inflammatory Effect of Abutilon crispum L. Medik Methanol Extract
}

\author{
Stephanie Triseptya Hunto, ${ }^{1}$ Kon Kuk Shin, ${ }^{1}$ Han Gyung Kim, ${ }^{1}$ Sang Hee Park, \\ Junsang Oh, ${ }^{2}$ Gi-Ho Sung, ${ }^{2}$ Mohammad Amjad Hossain, ${ }^{3}$ Ho Sik Rho, ${ }^{4}$ Jongsung Lee $\mathbb{D}^{1}$, \\ Jong-Hoon Kim $\mathbb{D}^{3},{ }^{3}$ and Jae Youl Cho $\mathbb{D D}^{1}$ \\ ${ }^{1}$ Department of Integrative Biotechnology, Sungkyunkwan University, Suwon 16419, Republic of Korea \\ ${ }^{2}$ Institute for Healthcare and Life Science, International St. Mary's Hospital and College of Medicine, Catholic Kwandong University, \\ Incheon 22711, Republic of Korea \\ ${ }^{3}$ Department of Veterinary Physiology, College of Medicine, Chonbuk National University, Iksan 54596, Republic of Korea \\ ${ }^{4}$ Department of Chemical Engineering, College of Engineering, Suwon University, Suwon 18323, Republic of Korea
}

Correspondence should be addressed to Jongsung Lee; bioneer@skku.edu, Jong-Hoon Kim; jhkim1@jbnu.ac.kr, and Jae Youl Cho; jaecho@skku.edu

Received 8 August 2018; Accepted 18 November 2018; Published 26 November 2018

Academic Editor: Maria T. Cruz

Copyright (C) 2018 Stephanie Triseptya Hunto et al. This is an open access article distributed under the Creative Commons Attribution License, which permits unrestricted use, distribution, and reproduction in any medium, provided the original work is properly cited.

\begin{abstract}
Abutilon crispum L. Medik, better known as bladdermallow, is used as a traditional remedy in India, for its anti-inflammatory effect due to its high content of flavonoids. However, research about its anti-inflammatory effect at the molecular level has not been performed. In this study, we aimed to investigate the mechanism of Abutilon crispum methanol extract (Ac-ME) in inhibiting the inflammatory response by conducting several experiments including cellular and molecular assays. Ac-ME inhibited the production of nitric oxide (NO) in RAW264.7 cells during treatment of LPS and Pam3CSK4 without exhibiting cytotoxicity. AcME also suppressed the mRNA expression of inducible nitric oxide (iNOS) and proinflammatory cytokines such as interleukin (IL) $-1 \beta$ and IL-6. Moreover, Ac-ME was shown to inhibit the NF- $\kappa$ B pathway, according to the luciferase reporter gene assay performed with a NF- $\kappa \mathrm{B}-$ Luc construct containing NF- $\kappa$ B-binding promoter regions under MyD88 and TRIF overexpression conditions, and immunoblotting analysis by determining the phospho-form levels of $\mathrm{I} \kappa \mathrm{B} \alpha, \mathrm{IKK} \alpha / \beta$, and p85, a regulatory domain of phosphatidylinositide 3-kinase (PI3K). Finally, we observed that the level of phospho-p85 induced by the overexpression of spleen tyrosine kinase (Syk) and Src was decreased by Ac-ME at $200 \mu \mathrm{g} / \mathrm{ml}$. Therefore, these results suggest that Ac-ME has an anti-inflammatory effect by targeting PI3K in the NF- $\kappa$ B signaling pathway.
\end{abstract}

\section{Introduction}

When the body is invaded by foreign organisms and toxins, it will provide protection in the form of an immune system response. The innate immune system is the first to react rapidly and perform an inflammatory response $[1,2]$, characterized by redness, swelling, and pain. The inflammatory cascade begins when the signature components of pathogens, known as pathogen-associated molecular patterns (PAMPs), interact with pattern recognition receptors (PRRs). Tolllike receptors (TLRs), one of the subclasses of PRRs, can recognize specific PAMPs from viruses, bacteria, fungi, and protozoa. For instance, TLR 2 recognizes peptidoglycans from gram positive bacteria, while TLR 4 recognizes LPS from gram-negative bacteria. The interactions of TLRs and PAMPs lead to recruitment of adaptor proteins such as MyD88, TRIF, TRAM, and TIRAP, which can mediate another cascade effect $[3,4]$. This cascade effect involves 
the signaling pathway leading to activation of transcription factors. The signal mainly consists of NF- $\kappa \mathrm{B}, \mathrm{AP}-1$, and IRF-3 pathways. The $\mathrm{NF} \kappa \mathrm{B}$ pathway is activated when the cascade passes through protein tyrosine kinases (Syk and $\mathrm{Src}$ ), serine/threonine kinases (AKT), phosphatidylinositide 3 -kinases $(\mathrm{PI} 3 \mathrm{~K})$, and $\mathrm{I} \kappa \mathrm{B}$ kinases $(\mathrm{IKK})$. It is called the NF$\kappa \mathrm{B}$ pathway due to involvement of the NF- $\kappa \mathrm{B}$ protein (the transcription factor), which is translocated to the nucleus. Translocation of this transcriptional factor will eventually lead to production of inflammatory genes (iNOS and other cytokines) and the release of proinflammatory mediators (NO, interleukins (IL-1), TNF- $\alpha$, and $\mathrm{PGE}_{2}$ ) [5].

Even though inflammation is important for the host defense against infections, it also may contribute to many chronic diseases. Tissue injury, oxidative stress, angiogenesis, and fibrosis are the results of a series of inflammatory responses that may lead to other deadly complications such as cancer $[6,7]$. Therefore, development of new treatment to prevent and treat these diseases is essential. Herbal remedies are becoming popular for treating inflammatory diseases, but some of them remain untested. Abutilon crispum L. Medik, better known as Herissantia crispa L. Brizicky, is a trailing perennial shrub of the family Malvaceae and is known in America as bladdermallow. Traditionally, it is used in India for treatment of asthma, cough, diabetes, ulcers, and jaundice [8]. This plant is rich in flavonoids but has not been well studied with regard to pharmacology [9]. One of the studies found that Abutilon crispum L. Medik can be used as an anti-inflammatory agent through in vivo experiments, but the exact mechanism remains unknown [10]. Therefore, we aimed to identify the molecular target of the methanolic extract of Abutilon crispum L. Medik with a special focus on the NF- $\kappa \mathrm{B}$ signaling pathway.

\section{Materials and Methods}

2.1. Materials. The methanol extract (code no: PBID 10699) of Abutilon crispum L. Medik (Ac-ME) was purchased from International Biological Material Research Centre (http://www.ibmrc.re.kr, Daejeon, Korea). Pam3CSK4, lipopolysaccharide (LPS, E. coli 0111:B4), 3-(4,5-dimethylthiazol2-yl)-2,5-diphenyltetrazolium bromide (MTT), polyethyleneimine (PEI), sodium dodecyl sulfate (SDS), and dimethyl sulfoxide (DMSO) were purchased from Sigma Chemical Co. (St. Louis, MO, USA). PP2 and Piceatannol (Picea) were purchased from Calbiochem (La Jolla, CA, USA). Fetal bovine serum (FBS), penicillin/streptomycin, TRIzol, DMEM, and RPMI 1640 were obtained from GIBCO (Grand Island, NY, USA). RAW264.7 (ATCC No.: CRL-2278) and HEK293 (ATCC No.: CRL-1573) cells were products from American Type Culture Collection (Rockville, MD, USA). Primers used for semiquantitative reverse transcriptase polymerase chain reactions (RT-PCR) were obtained from Macrogen Inc. (Seoul, Korea). Luciferase constructs with NF- $\kappa$ B binding sites and epitope-tagged signaling expression constructs (FLAG-MyD88, CFP-TRIF, HA-Src, and Myc-Syk) were used as previously reported [11]. All other chemicals were Sigma grade. Phospho-specific and total antibodies recognizing the
NF- $\kappa$ B family were obtained from Cell Signaling Technology (Beverly, MA, USA).

2.2. Cell Culture and Drug Preparation. RAW264.7 cells were maintained in RPMI 1640 supplemented with $10 \%$ heat-inactivated FBS and $1 \%$ penicillin/streptomycin, while HEK293 cells were cultured in DMEM media complemented with $5 \%$ heat-inactivated FBS and $1 \%$ penicillin/streptomycin at $37^{\circ} \mathrm{C}$ in $5 \% \mathrm{CO}_{2}$. For each experiment, RAW264.7 and HEK293 cells were detached with cell scrappers and trypsin, respectively. The cells were counted with Trypan blue dye exclusion tests to achieve the desirable experimental cell density. Stock solutions of Ac-ME, Pam3CSK4, PP2, and piceatannol were prepared in DMSO for the in vitro experiments.

2.3. HPLC Analysis of Ac-ME. The constituents of Abutilon crispum L. Medik in methanol extract were analyzed using high-performance liquid chromatography (HPLC), as reported previously [12]. The standard compounds used in this analysis were quercetin, luteolin, and kaempferol. The conditions used in the HPLC analysis are described in Table 1 .

2.4. Measurement of NO Production. RAW264.7 macrophage cells were maintained for 18 hours, pretreated with Ac-ME (0$200 \mu \mathrm{g} / \mathrm{ml})$ for 30 minutes, and further incubated with LPS $(1 \mu \mathrm{g} / \mathrm{ml})$ for 24 hours. The inhibitory effects of Ac-ME on production of $\mathrm{NO}$ were determined by interpreting the NO level using Griess reagents and measured using a Synergy HT Multi-Mode Microplate Reader (BioTek Instruments $\mathrm{GmbH}$, Bad Friedrichshall, Germany) as previously reported [13].

2.5. Cell Viability Test. RAW264.7 or HEK293 cells were plated with a density of $1 \times 10^{6}$ cells $/ \mathrm{ml}$, preincubated for 18 hours, and then treated with Ac-ME (0-200 $\mu \mathrm{g} / \mathrm{ml})$ for an additional 24 hours. The cytotoxic effect of Ac-ME was tested by a conventional MTT assay, as previously reported [14, 15]. MTT solution $(10 \mathrm{mg} / \mathrm{ml}$ in phosphate-buffered saline, $\mathrm{pH}$ $7.4,5 \mu \mathrm{g} / \mathrm{ml}$ as a final concentration) was added, and the cells were continuously cultured for three hours. The incubation was terminated by the addition of $15 \%$ SDS into each well, solubilizing the formazan that was then measured using a Synergy HT Multi-Mode Microplate Reader with $570 \mathrm{~nm}$ absorbance $\left(\mathrm{OD}_{570-630}\right)$.

2.6. mRNA Precipitation and Analysis Using Semiquantitative Reverse Transcriptase Polymerase Chain Reactions. RAW264.7 cells were plated with a density of $1 \times 10^{6}$ cells $/ \mathrm{ml}$, preincubated for $18 \mathrm{~h}$, and then treated with Ac-ME (0$200 \mu \mathrm{g} / \mathrm{ml})$ in the presence or absence of LPS $(1 \mu \mathrm{g} / \mathrm{ml})$ for $6 \mathrm{~h}$. Total RNA was isolated from the RAW264.7 cells using TRIzol reagent according to the manufacturer's instructions, and $1 \mu \mathrm{g}$ of total RNA was used for cDNA synthesis with a cDNA synthesis kit (Thermo Fisher Scientific, Waltham, MA, USA). Total RNA was stored at $-70^{\circ} \mathrm{C}$ until use. Semiquantitative RT-PCR reactions were conducted as previously 
TABLE 1: HPLC condition for analyzing Ac-ME constituents with quercetin, luteolin, and kaempferol as standard compounds.

\begin{tabular}{|c|c|c|c|}
\hline Instrument & \multicolumn{3}{|c|}{ KNAUER Corp. HPLC system } \\
\hline Column & \multicolumn{3}{|c|}{ Phenomenex, Gemini $5 \mu \mathrm{m} \mathrm{C18} \mathrm{100A,} 250 \times 4.60 \mathrm{~mm} 5 \mathrm{~mm}$} \\
\hline Column temperature & \multicolumn{3}{|c|}{$35^{\circ} \mathrm{C}$} \\
\hline Detector & \multicolumn{3}{|c|}{ UV/VIS detector $370 \mathrm{~nm}$} \\
\hline Analysis software & \multicolumn{3}{|c|}{ ClarityChrom Software } \\
\hline Injection volume & \multicolumn{3}{|c|}{$20 \mu \mathrm{l}$} \\
\hline Standard & \multicolumn{3}{|c|}{ Diluted with DMSO } \\
\hline Sample treatment & \multicolumn{3}{|c|}{$50 \mathrm{mg} / \mathrm{ml}$, diluted with DMSO } \\
\hline \multicolumn{4}{|l|}{ Mobile phase } \\
\hline Solvent A & \multicolumn{3}{|c|}{$0.1 \%$ TFA in $\mathrm{H}_{2} \mathrm{O}$} \\
\hline Solvent B & \multicolumn{3}{|c|}{$0.08 \% \mathrm{TFA}$ in $95 \% \mathrm{MeCN}+5 \% \mathrm{H}_{2} \mathrm{O}$} \\
\hline Flow rate & \multicolumn{3}{|c|}{$1.0 \mathrm{ml} / \mathrm{min}$} \\
\hline \multirow[t]{4}{*}{ Time table } & Time (min) & Solvent A (\%) & Solvent B(\%) \\
\hline & 0 & 100 & 0 \\
\hline & 50 & 50 & 50 \\
\hline & 60 & 0 & 100 \\
\hline
\end{tabular}

TABle 2: Primer sequences used in PCR analysis.

\begin{tabular}{|c|c|c|}
\hline Gene Target & & Sequence $\left(5^{\prime}-3^{\prime}\right)$ \\
\hline \multirow[t]{2}{*}{ iNOS } & $\bar{F}$ & GTG AAG AAA ACC CCT TGT GCT G \\
\hline & $\mathrm{R}$ & AGT TCC GAG CGT CAA AGA CC \\
\hline \multirow[t]{2}{*}{ IL-1 $\beta$} & $\mathrm{F}$ & CAG GAT GAG GAC ATG AGC ACC \\
\hline & $\mathrm{R}$ & CTC TGC AGA CTC AAA CTC CAC \\
\hline \multirow[t]{2}{*}{ IL-6 } & $\mathrm{F}$ & GCC TTC TTG GGA CTG ATG CT \\
\hline & $\mathrm{R}$ & TGG AAA TTG GGG TAG GAA GGA C \\
\hline \multirow[t]{2}{*}{ GADPH } & $\mathrm{F}$ & ACC ACA GTC CAT GCC ATC AC \\
\hline & $\mathrm{R}$ & CCA CCA CCC TGT TGC TGT AG \\
\hline
\end{tabular}

reported [16]. All the primers used in this study are shown in Table 2.

\subsection{Plasmid Transfection and Luciferase Reporter Gene Assay.} RAW264.7 and HEK293 cells ( $1 \times 10^{6}$ cells/ml) were transfected with $1.1 \mu \mathrm{g}$ of plasmid encoding $\beta$-galactosidase and $\mathrm{NF}-\kappa \mathrm{B}-\mathrm{luc}$ in the presence or absence of an inducing molecule (Flag-MyD88, CFP-TRIF, Myc-Syk, or HA-Src) using the PEI method for 24 hours. The cells were then treated with Ac-ME at the indicated concentration for 24 hours. The cell aliquots were treated with either $\beta$-galactosidase or luciferase buffer to measure the absorbance and luminescence, respectively. Luciferase assays were conducted using the Luciferase Assay System (Promega, Madison, WI, USA) as previously reported $[11,17]$.

2.8. Preparation of Total Lysates and Immunoblotting. RAW264.7 or HEK293 cells were plated with a density of 2 $\times 10^{6}$ cells $/ \mathrm{ml}$, preincubated for $18 \mathrm{~h}$, and then treated with Ac-ME (100 or $200 \mu \mathrm{g} / \mathrm{ml})$ in the presence or absence of LPS $(1 \mu \mathrm{g} / \mathrm{ml})$ for indicated times. Ac-ME-treated RAW264.7 and HEK293 cells were harvested, washed in cold PBS, and lysed in lysis buffer [20 mM Tris- $\mathrm{HCl}$ (pH 7.4), $20 \mathrm{mM} \mathrm{NaF}$, $2 \mathrm{mM}$ EDTA, $2 \mathrm{mM}$ EGTA, $50 \mathrm{mM} \beta$-glycerol phosphate,
$1 \mathrm{mM}$ DTT, $2 \mu \mathrm{g} / \mathrm{ml}$ aprotinin, $2 \mu \mathrm{g} / \mathrm{ml}$ leupeptin, $1 \mu \mathrm{g} / \mathrm{ml}$ pepstatin, $50 \mathrm{mM}$ PSMF, $1 \mathrm{mM}$ benzamide, $2 \%$ triton $\mathrm{X}$ $100,10 \%$ glycerol, $0.1 \mathrm{mM}$ sodium vanadate, and $1.6 \mathrm{mM}$ pervanadate] for 30 minutes. The lysates were then cleared by centrifugation at $12000 \mathrm{xg}$ for 10 minutes at $4^{\circ} \mathrm{C}$, and the supernatants were stored at $-20^{\circ} \mathrm{C}$. The whole cells were analyzed using immunoblotting as previously reported [18]. Densitometric scanning values of each protein from blots were obtained using the DNR Bio-imaging system (a Gelquant software Ver. 2.7, Neve Yamin, Israel). Calculation of relative intensity was carried out with the following equation. Relative intensity $=$ densitometric scanning value of phosphoproteins or total proteins/densitometric scanning value of corresponding total protein or loading control ( $\beta$-actin).

2.9. Statistical Analysis. Data are expressed as mean \pm standard deviation (SD) of at least three independent experiments performed in triplicate or of one representative experiment out of three different experiments with similar results. The results were interpreted using either ANOVA/Scheffe's post hoc test or the Kruskal-Wallis/Mann-Whitney test for statistical comparisons. The data was statistically significantly different if $\mathrm{p}<0.05$. All statistical tests were carried out using the computer program SPSS (SPSS Inc., Chicago, IL, USA). 


\section{Results}

3.1. Effect of Ac-ME on Inflammatory Responses. To investigate whether Ac-ME has an effect on modulating the inflammatory response, the NO (nitric oxide) level of the LPS-treated RAW264.7 cells (TLR 4-ligand) was determined. As shown in Figure 1(a) left panel, Ac-ME dose-dependently decreased the level of NO production to $48.62 \pm 0.99 \%$ at $200 \mu \mathrm{g} / \mathrm{ml}$. Similarly, it also diminished the production of NO from Pam3CSK4-treated RAW264.7 cells (TLR 2-Ligand) to $5.97 \pm 1.07 \%$ at $200 \mu \mathrm{g} / \mathrm{ml}$. Standard anti-inflammatory compounds such as prednisolone demonstrated similar activity in inhibiting NO level. In Figure 1(a) right panel, prednisolone dose-dependently suppressed the NO level to $31.68 \pm 15.74 \%$ at $400 \mu \mathrm{g} / \mathrm{ml}$. From Figures 1(b) left panel and 1(b) right panel, no cell toxicity was observed in the RAW264.7 and HEK293 cells, indicating that the NO inhibitory effects were not due to the toxic activity of the compounds.

Ac-ME were analyzed using HPLC analysis to identify its anti-inflammatory constituents. For this, we selected quercetin, luteolin, and kaempferol, because not only these compounds were found to suppress macrophage-mediated inflammatory responses [19-21], but also these compounds were identified in Genus Abutilon [22]. As shown in Figure 1(c) left panel, the components of Ac-ME were analyzed with the standard anti-inflammatory compounds quercetin, luteolin, and kaempferol. In Figure 1(c) right panel, the HPLC data showed that Ac-ME contains $0.098 \%$ of kaempferol, $0.006 \&$ of quercetin, and $0.002 \%$ of luteolin.

3.2. Ac-ME Suppresses Inflammatory Responses at the Transcriptional Level. mRNA levels were examined to determine whether Ac-ME can modulate inflammation at the transcriptional or translational level. As seen in Figure 2(a), LPS treatment increased the levels of iNOS, COX-2, TNF- $\alpha$, IL- $1 \beta$, and IL-6. Ac-ME suppressed the upregulation level of iNOS, IL-6, and IL-1 $\beta$ at $200 \mu \mathrm{g} / \mathrm{ml}$, which indicates an effect on transcription. The regulatory role of Ac-ME on the activation of inflammatory transcription factors was determined by the luciferase assay. HEK293 cells were cotransfected with myeloid differentiation primary response 88 (MyD88) and TIR-domain-containing adapter-inducing interferon- $\beta$ (TRIF) together with NF- $\kappa \mathrm{B}$ luciferase gene. Treatment of Ac-ME to the transfected cells inhibited the NF- $\kappa$ B mediated luciferase activity in a dose-dependent manner up to $20.43 \%$ and $66.89 \%$ at $200 \mu \mathrm{g} / \mathrm{ml}$, respectively, shown in Figure 2(b) left panel and right panel.

3.3. Ac-ME Inhibits the NF- $\kappa B$ Activation Pathway. To identify the exact target of Ac-ME, we conducted immunoblotting analysis to determine which proteins are stopped in the cascade of the inflammatory response. As we expected and as shown in Figure 3(a) (left and right panels), Ac-ME $(200 \mu \mathrm{g} / \mathrm{ml})$ clearly suppressed the level of $\mathrm{p}-\mathrm{I} \kappa \mathrm{B} \alpha$ upregulated by LPS at 5, 15,30, and $60 \mathrm{~min}$. Through the analysis of upstream signaling, it was found that the phosphorylation of p85/PI3K was significantly blocked by this extract at 5,15 , 30 , and 60 minutes. Overexpression of its upstream kinases
(Syk and Src) was performed to observe the involvement of these two proteins in Ac-ME-mediated anti-inflammatory action. Ac-ME did not inhibit either Src or Syk, but as shown in Figures 3(b) and 3(c) left panel and right panel, p-PI3K was blocked by Ac-ME both in Src and Syk overexpression. Finally, NO production was also strongly diminished by specific inhibitors (PP2 and Piceatannol) of Src and Syk, indicating that these enzymes play critical roles in inflammatory responses in LPS-treated macrophages (Figure 3(d)).

\section{Discussion}

Natural remedies are becoming popular in the pharmaceutical field and market due to the desire for fewer synthetic ingredients and side effects. Therefore, the development of plant-derived drugs is preferable in the research field nowadays. Abutilon crispum L. Medik is one of the plants whose function has yet to be widely known. However, some papers previously reported that Abutilon crispum has an anti-inflammatory effect [10], but the molecular mechanism behind it has not been revealed. Therefore, in this study, we investigated the anti-inflammatory effect of Ac-ME regarding the NF- $\kappa$ B pathway.

First, we performed the NO assay to determine whether Ac-ME can inhibit the production of inflammatory mediators. NO or nitric oxide is one of the inflammatory mediators or signaling molecules that can induce inflammation when overproduced [23]. To induce NO production, LPS and Pam3CSK 4 were used as inducers in the NO assay. LPS and Pam3CSK 4 are molecule-derived pathogens or PAMPs that can be recognized by TLRs and activate the signaling cascade to produce inflammatory cytokines and a resulting inflammatory response [24]. LPS or lipopolysaccharide is derived from the outer surface membrane component in gramnegative bacteria and induces a strong innate immunity in humans [25], while Pam3CSK4 is a synthetic tripalmitoylated lipopeptide that can mimic the acetylated amino terminus of bacterial lipoproteins and can be recognized by TLR 2 [26]. From the results shown in Figure 1(a), Pam3CSK4 and LPS can dose-dependently downregulate the production of NO treated by Ac-ME. To confirm the pattern of Ac-ME inhibiting NO production, we also assessed an anti-inflammatory drug, prednisolone. Prednisolone is a corticosteroid drug that suppresses inflammatory genes by reversing histone acetylation-activated inflammatory genes through the binding of liganded glucocorticoid receptors (GR) to coactivators and recruitment of histone deacetylase-2 (HDAC2) to the activated transcription complex [27]. Figure 1(b) shows that the prednisolone group dose-dependently inhibited the level of NO production. Prednisolone exhibits the same pattern in downregulating the level of NO production as Ac-ME. Therefore, from the NO data, we conclude that Ac-ME has antiinflammatory activity. We also must confirm that this result is not due to the toxicity of the compound to the cell. As a result, we assessed cell viability by performing the MTT assay with RAW264.7 and HEK293 cells. In the assay, the compound (Figure 1(b) (left panel)) did not exhibit any toxicity in either cell lines or the group treated with prednisolone (Figure 1(b) 

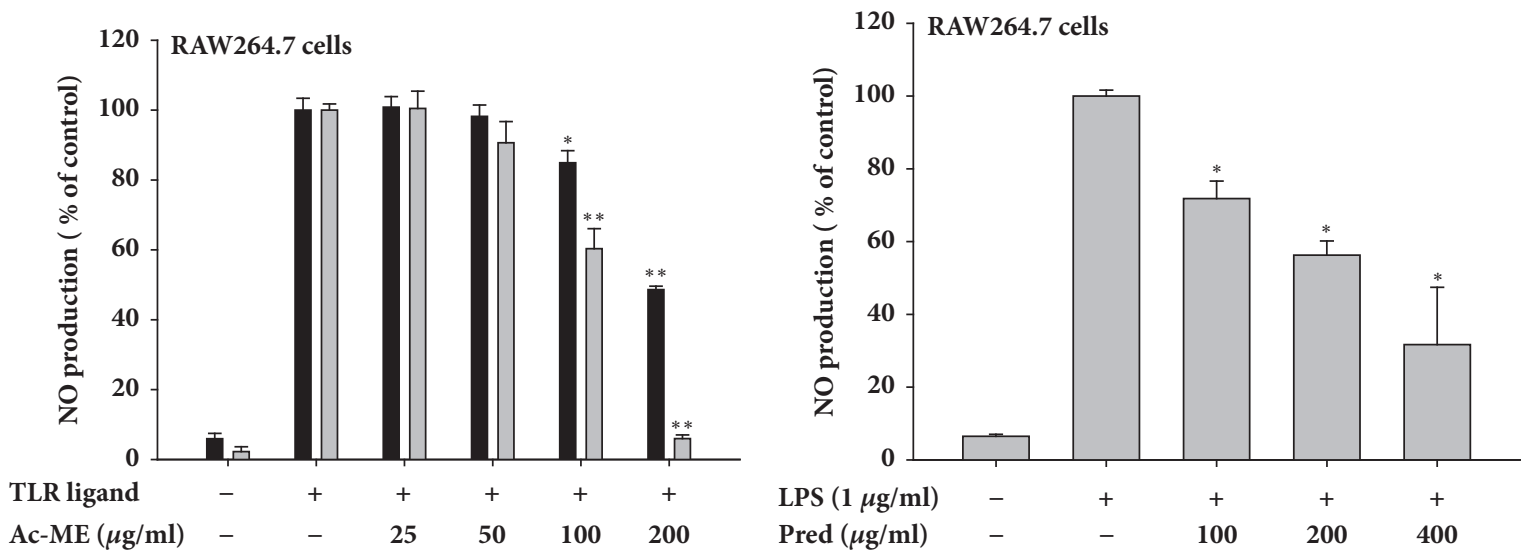

(a)
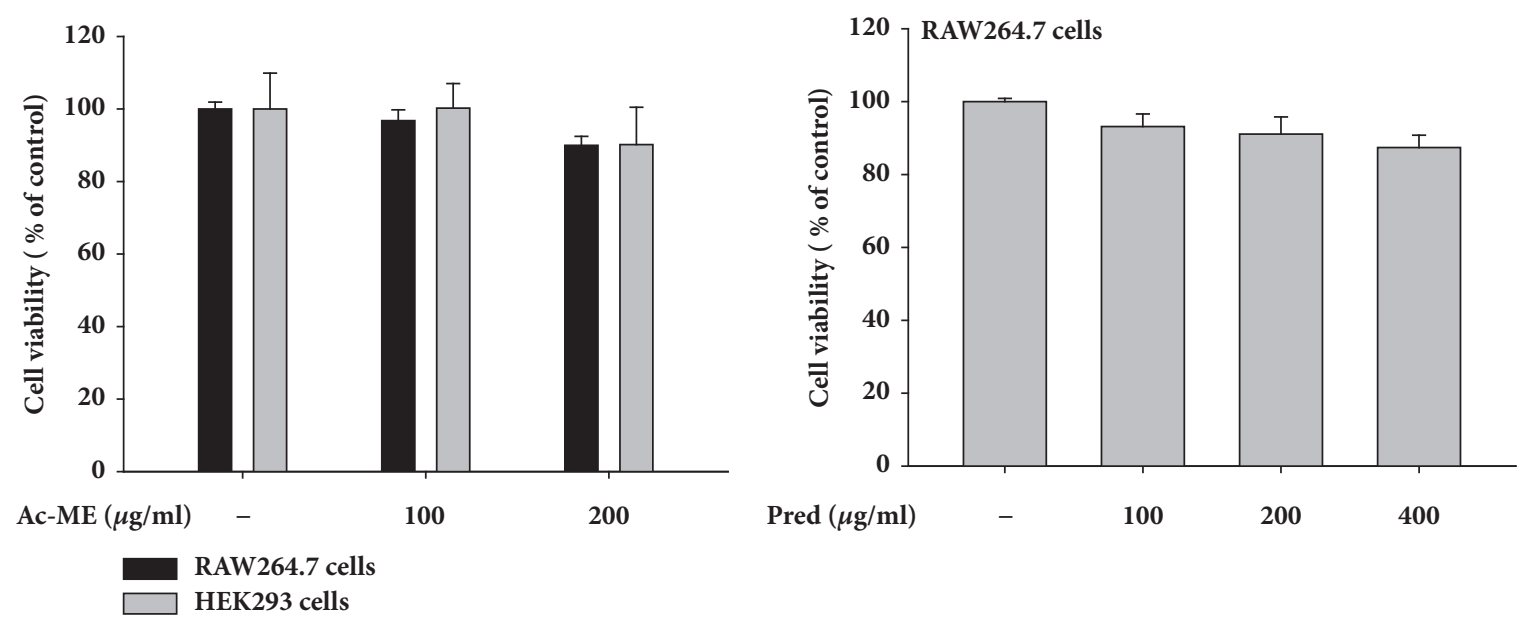

(b)
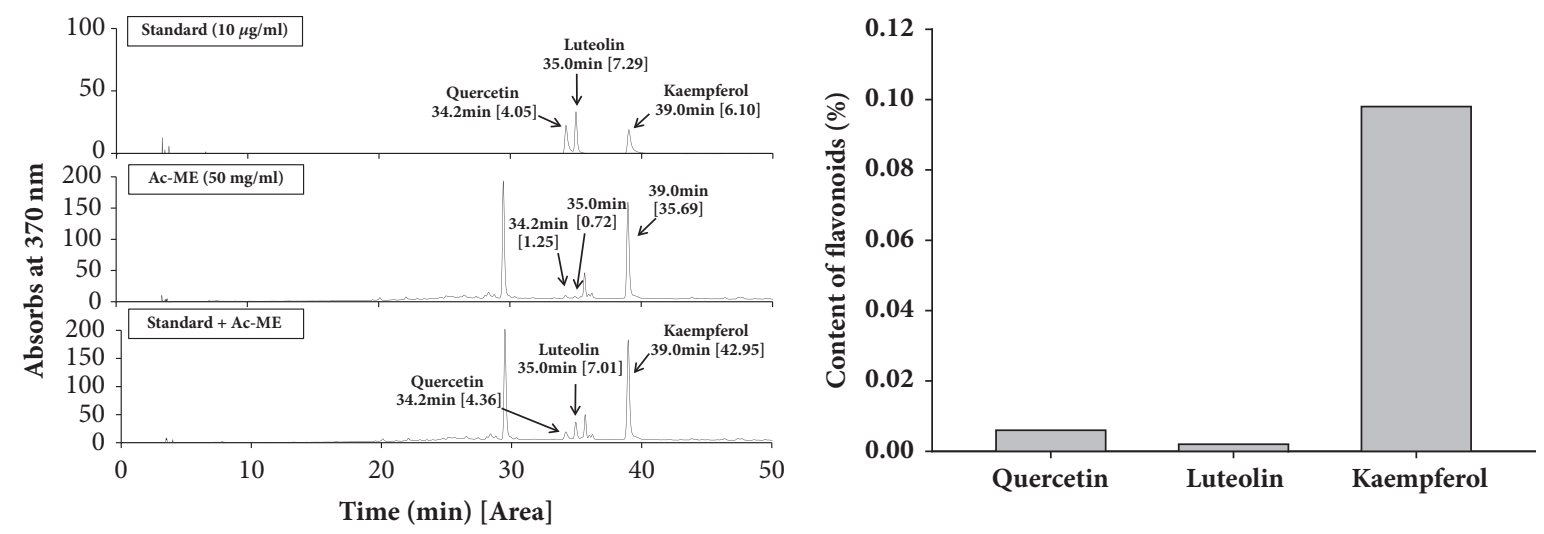

(c)

FIGURE 1: Effect of Ac-ME on production of inflammatory mediators and HPLC profiling of Ac-ME. (a) Levels of NO production induced by Pam3CSK4 (a TLR2 ligand) and LPS (a TLR 4 ligand) were determined by the Griess Assay from supernatant of RAW264.7 cell treated with Ac-ME (left panel) or prednisolone (Pred) (right panel) for $24 \mathrm{~h}$. (b) Cell viability of RAW264.7 and HEK293 cells treated with Ac-ME (left panel) or Pred (right panel) was determined by MTT assay. (c) The phytochemical profile of Ac-ME was analyzed by HPLC with the conditions described in Table 1 (left panel). The content of flavonoids was shown in the graph by calculating from the standard curves of the compounds (right panel). $* P<0.05$ and $* * P<0.01$ compared to the control. 

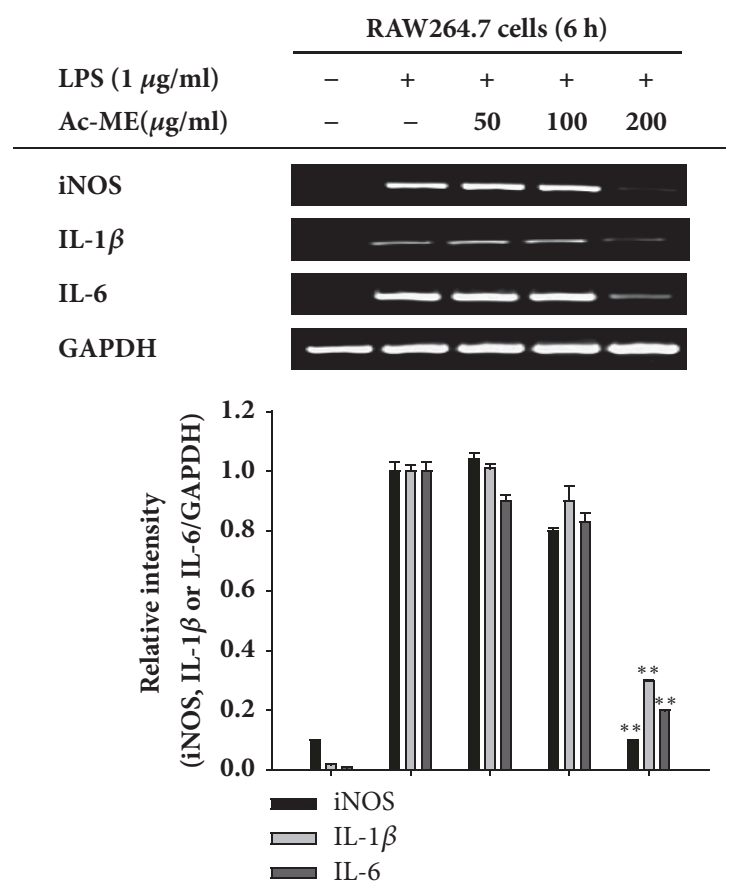

(a)
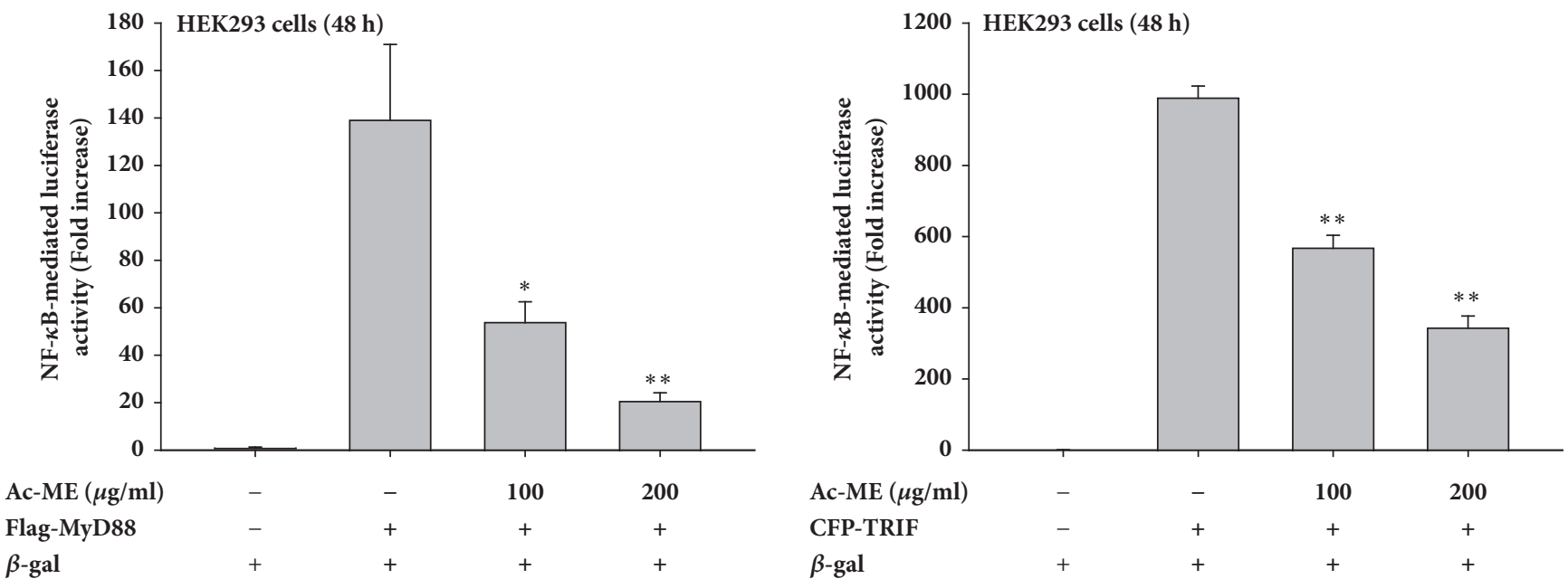

(b)

FIGURE 2: Effect of Ac-ME on the transcriptional regulation of inflammatory genes. (a) Using semiquantitative RT-PCR, the mRNA levels of iNOS, IL-1 $\beta$, and IL-6 were determined (upper panel). Relative intensity (lower panel) was values of ratio calculated using densitometric scanning values of inflammatory genes (iNOS, IL-1 $\beta$ or IL-6) and densitometric scanning values of GAPDH by the DNR Bio-imaging system (a Gelquant software Ver. 2.7). (b) HEK293 cells were cotransfected with MyD88 (left panel) and TRIF (right panel). The luciferase activity was measured using a luminometer. $* P<0.05$ and $* * P<0.01$ compared to the control.

(right panel)). We also attempted to identify the components in Ac-ME which contribute to its anti-inflammatory activity. HPLC analysis was done with standard compounds of antiinflammatory flavonoids including luteolin, quercetin, and kaempferol [28]. In Figure 1(c) right panel, it is shown that kaempferol has the highest content among others. So far, few information of phytochemical feature of this plant has been revealed. Thus, it was reported that $\beta$-sitosterol, lupeol, and $\beta$-amyrin are contained in the leaves of this plant [29]. In addition, genus Abutilon has been reported to contain flavonoids, phenolic acids, sterols, triterpenes, coumarins, alkaloids, lactones, megastigmanes, and iridoids [22]. With kaempferol that we have identified in this study, a lot of compounds including caffeic acid, rutin, myricetin, catechin, benzoic acid, $\beta$-amyrin, lupeol, and $\beta$-sitosterol have been known as anti-inflammatory components [3032]. Therefore, some of these compounds included in Ac-ME could contribute to the anti-inflammatory activity of Ac-ME. 


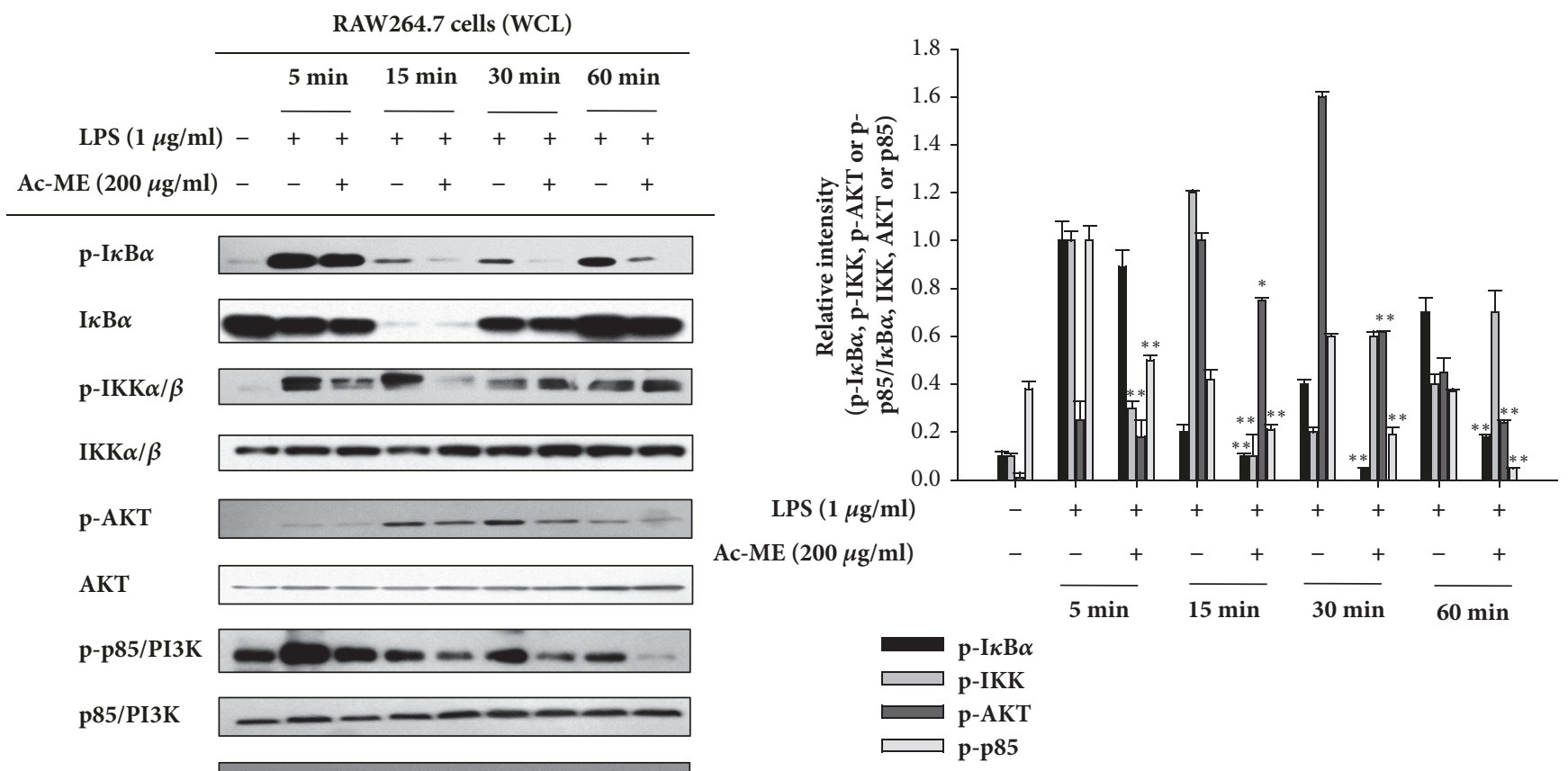

$\beta$-actin

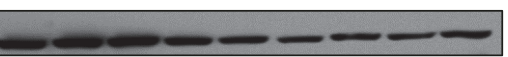

(a)

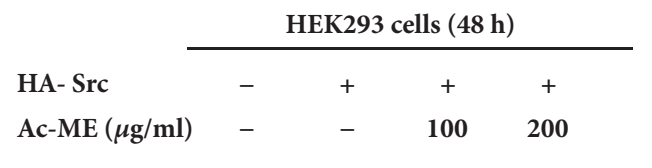

HA

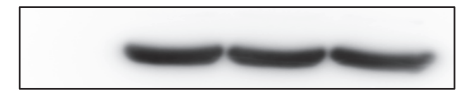

p-p85/PI3K

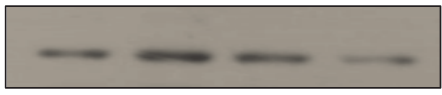

$\beta$-actin

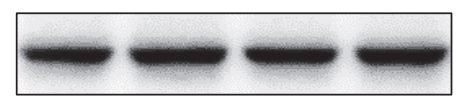

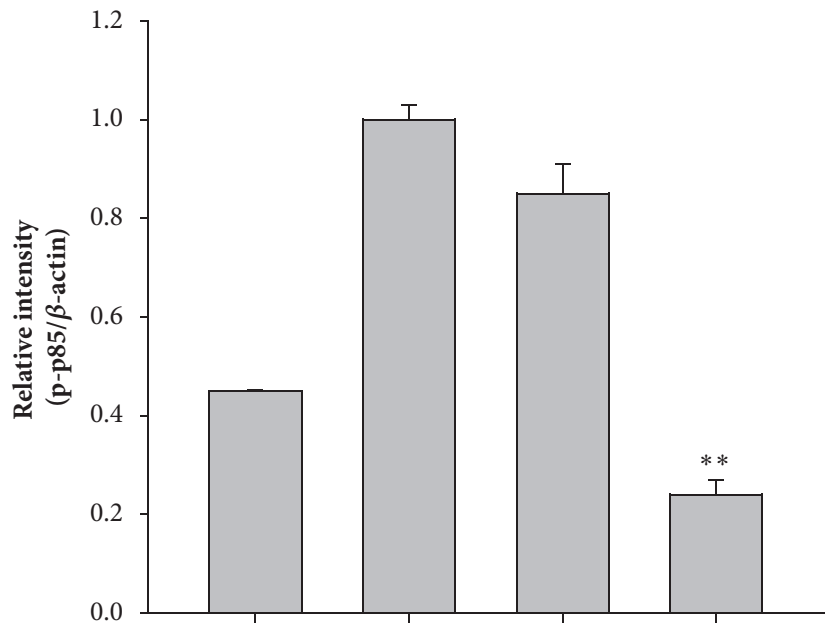

(b)
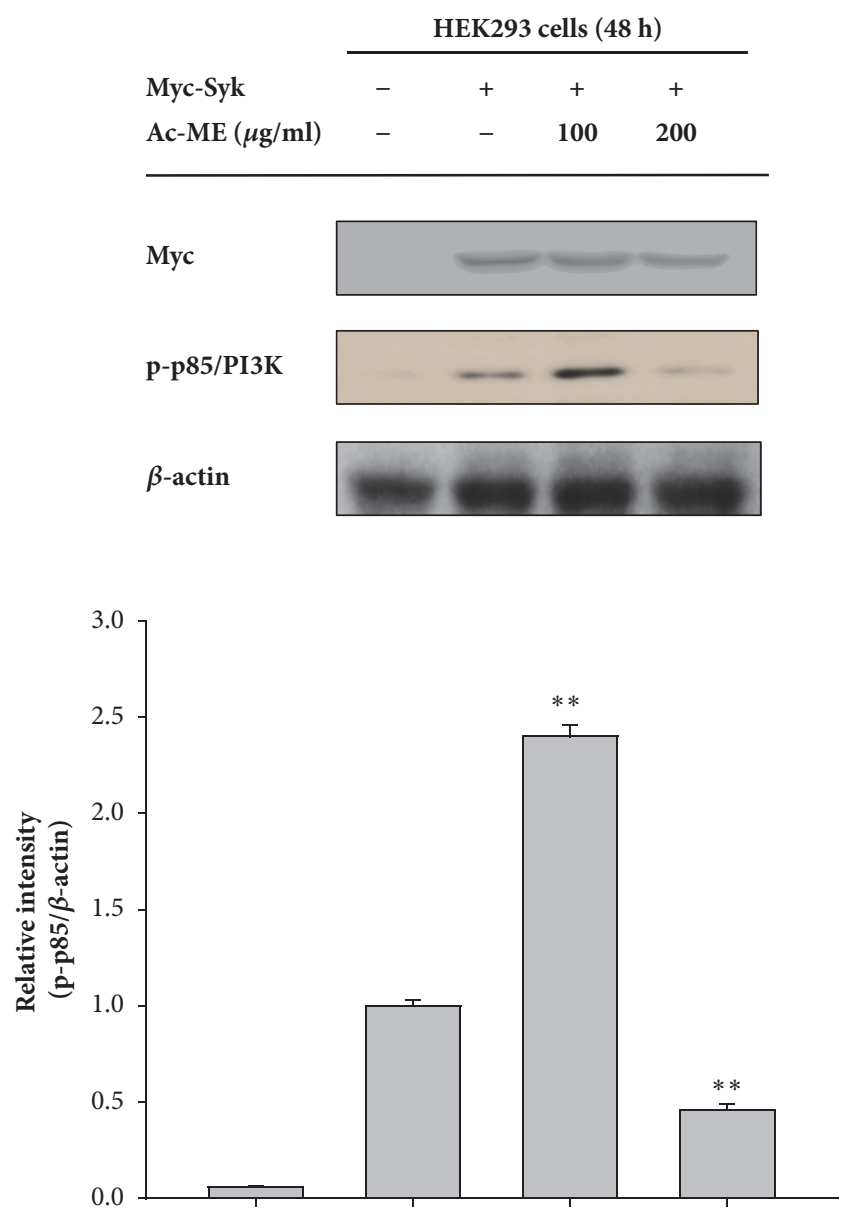

(c)

FIgURE 3: Continued. 


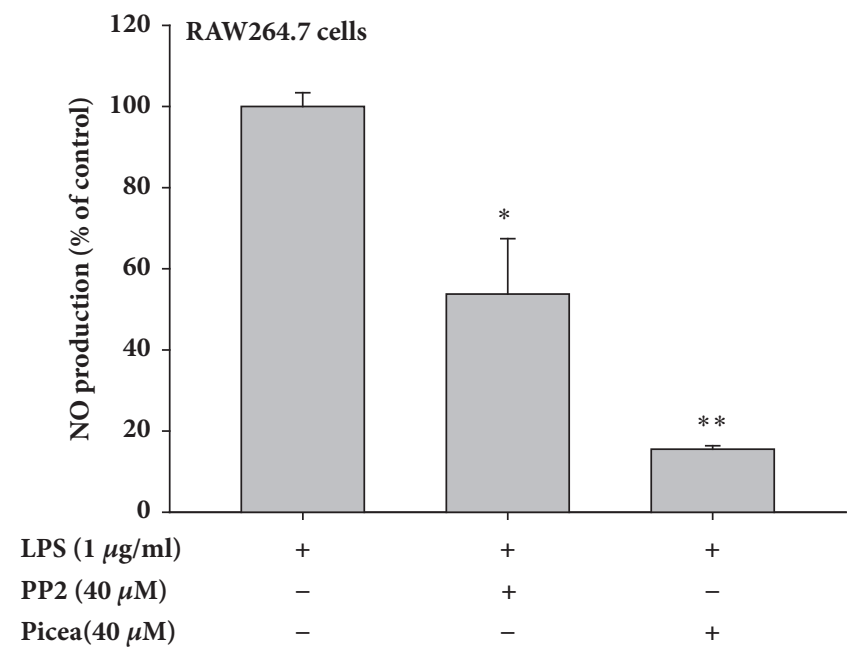

(d)

FIGURE 3: Effect of Ac-ME on the upstream signaling of the NF- $\kappa$ B pathway. Immunoblotting was performed to determine the target of AcME in modulating the inflammatory response. ((a) left panel) Phosphoprotein and total protein levels of $\mathrm{I} \kappa \mathrm{B} \alpha, \mathrm{IKK} \alpha / \beta, \mathrm{AKT}$, and $\mathrm{p} 85 / \mathrm{PI} 3 \mathrm{~K}$ were determined by immunoblotting analysis with specific antibodies. ((a) right panel) Relative intensity was values of ratio calculated using densitometric scanning values of $\mathrm{p}-\mathrm{I} \kappa \mathrm{B} \alpha, \mathrm{p}-\mathrm{IKK} \alpha / \beta, \mathrm{p}-\mathrm{AKT}$, or $\mathrm{p}-\mathrm{p} 85$ and densitometric scanning values of their total proteins (I $\kappa \mathrm{B} \alpha$, IKK $\alpha / \beta$, AKT, or p85) by the DNR Bio-imaging system (a Gelquant software Ver. 2.7). (b, c) HEK293 cells were transfected with Myc-Syk or HA-Src and, at $36 \mathrm{~h}$, Ac-ME $(100-200 \mu \mathrm{g} / \mathrm{ml})$ was treated to the transfected cells for further $12 \mathrm{~h}$. The levels of phospho-p85, $\beta$-actin, Myc, or HA were identified by immunoblotting analysis. Relative intensity was values of ratio calculated using densitometric scanning value of p-p85 and densitometric scanning value of $\beta$-actin by the DNR Bio-imaging system (a Gelquant software Ver. 2.7). (d) The NO inhibitory levels of Syk and Src inhibitors (PP2 and Piceatannol) were measured with LPS-treated RAW264.7 cells. $* P<0.05$ and $* * P<0.01$ compared to the control.

By activity-guided fractionation, we will further define additional anti-inflammatory constituents from Ac-ME in the following project.

When inflammation occurs, $\mathrm{NO}$ is produced by inducible nitric oxide synthase (iNOS) in the macrophages [33]. iNOS is responsible for the roles of $\mathrm{NO}$ in the inflammatory response [34]. Other genes also play an important role in interceding inflammation. Therefore, we analyzed the mRNA expression of inflammatory genes by performing a reverse transcriptase polymerase chain reaction (RT-PCR). iNOS, IL- $1 \beta$, and IL-6 were investigated with RT-PCR, and the expression of these genes were inhibited by Ac-ME at $200 \mu \mathrm{g} / \mathrm{ml}$, as shown in Figure 2(a) left panel and right panel.

To confirm whether Ac-ME is capable of modulating the activation of transcription factors, we performed the luciferase reporter gene assay. Cells were cotransfected with TRIF (TIR-domain containing adapter-inducing interferonb) and MyD88 (myeloid differentiation primary response), an adaptor protein that can induce NF- $\kappa \mathrm{B}$-mediated luciferase activity. When the TLR binds with its ligand, the receptor will recruit the adaptor protein so that the cascade will occur [35]. Figures 2(b) left panel and 2(b) right panel show that Ac-ME can alter NF- $\kappa$ B-mediated luciferase activity. Therefore, we continued to search for the target of Ac-ME upstream.

We performed immunoblotting assay with western blot analysis. Figure 3(a) shows that the phospho-p85, a regulatory subunit of PI3K, was inhibited by Ac-ME after 5 min of cells exposure to LPS. Therefore, we measured the overexpression strategies by overexpressing the tyrosine kinases, Syk and Src, in HEK293 cells. In Figures 3(b) and 3(c) left panel and right panel, both p-p85 proteins were inhibited by Ac-ME with the overexpression of Syk and Src. Figure 3(d) shows the inhibitory level of NO production when treated with PP2 and Piceatannol, inhibitors of Syk and Src, respectively. There was a significant difference between the control group and the inhibitor-treated group. These results strongly indicate that the anti-inflammatory effects of Ac-ME are mediated through the suppression of NF- $\kappa \mathrm{B}$ as a result of inhibition of PI3K (Figure 4). Further studies are required to understand how Ac-ME can inhibit PI3K in the inflammatory response and to discover other possible targets of Ac-ME.

\section{Conclusion}

The study showed that the Ac-ME has an anti-inflammatory effect in the NF- $\kappa$ B pathway by inhibiting the phosphorylation of $\mathrm{p} 85 / \mathrm{PI} 3 \mathrm{~K}$.
Abbreviations
HEK: Human embryonic kidney
COX: Cyclooxygenase
TNF- $\alpha$ : Tumor necrosis-alpha
IL: $\quad$ Interleukin
GAPDH: Glyceraldehyde 3-phosphate dehydrogenase
$\mathrm{I} \kappa \mathrm{B} \alpha: \quad$ Inhibitor of kappa $\mathrm{B}$ alpha
IKK: $\quad$ I $\kappa$ B kinase
NF- $\kappa$ B: $\quad$ Nuclear factor-kappa B
AP-1: Activator protein-1 


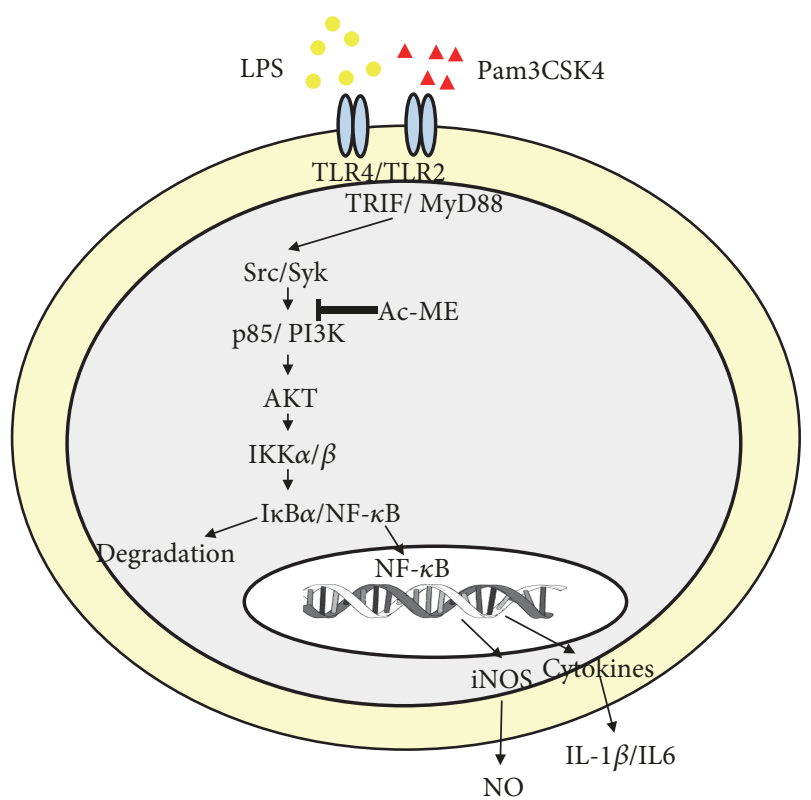

Figure 4: Putative inhibitory pathway of Ac-ME in mediating the inflammatory response. It is assumed that Ac-ME targets the activation of both Src and Syk linked to the activation of intracellular signaling pathway for the nuclear translocation of NF- $\kappa$ B. Suppression of this pathway leads to the downregulation of iNOS-mediated NO production and the expression of proinflammatory cytokines such as IL-1 $\beta$ and IL-6.

IRF-3: Interferon regulatory factor-3

Picea: Piceatannol

Pred: Prednisolone

FBS: Fetal bovine serum.

\section{Data Availability}

The data used to support the findings of this study are available from the corresponding author upon request.

\section{Conflicts of Interest}

The authors have no conflicts of interest to declare.

\section{Authors' Contributions}

Stephanie Triseptya Hunto, Jongsung Lee, Jong-Hoon Kim, and Jae Youl Cho designed the experiments. Stephanie Triseptya Hunto, Kon Kuk Shin, Han Gyung Kim, Sang Hee Park, Junsang Oh, Gi-Ho Sung, and Mohammad Amjad Hossain performed the laboratory assays. Stephanie Triseptya Hunto, Ho Sik Rho, Jongsung Lee, Jong-Hoon Kim, and Jae Youl Cho analyzed the data. Stephanie Triseptya Hunto, Jongsung Lee, Jong-Hoon Kim, and Jae Youl Cho wrote the manuscript. All authors read and approved the manuscript.

\section{Acknowledgments}

This research was supported by the Basic Science Research Program through the National Research Foundation of Korea (NRF) funded by the Ministry of Education, Republic of Korea (2017R1A6A1A03015642).

\section{References}

[1] K. Nasu and H. Narahara, "Pattern recognition via the toll-like receptor system in the human female genital tract," Mediators of Inflammation, vol. 2010, Article ID 976024, 12 pages, 2010.

[2] J. H. Kim, B. C. Yoo, W. S. Yang, E. Kim, S. Hong, and J. Y. Cho, "The Role of Protein Arginine Methyltransferases in Inflammatory Responses," Mediators of Inflammation, vol. 2016, Article ID 4028353, 11 pages, 2016.

[3] R. Medzhitov, "Toll-like receptors and innate immunity," Nature Reviews Immunology, vol. 1, no. 2, pp. 135-145, 2001.

[4] J. H. Kim, Y.-S. Yi, M.-Y. Kim, and J. Y. Cho, "Role of ginsenosides, the main active components of Panax ginseng, in inflammatory responses and diseases," Journal of Ginseng Research, vol. 41, no. 4, pp. 435-443, 2017.

[5] W. S. Yang, Y.-S. Yi, D. Kim et al., "Nuclear factor kappa-Band activator protein-1-mediated immunostimulatory activity of compound $\mathrm{K}$ in monocytes and macrophages," Journal of Ginseng Research, vol. 41, no. 3, pp. 298-306, 2017.

[6] P. Libby, "Inflammatory mechanisms: the molecular basis of inflammation and disease," Nutrition Reviews, vol. 65, no. 3, pp. S140-S146, 2007.

[7] N. Karachaliou, M. Gonzalez-Cao, G. Crespo et al., "Interferon gamma, an important marker of response to immune checkpoint blockade in non-small cell lung cancer and melanoma patients," Therapeutic Advances in Medical Oncology, vol. 10, p. 175883401774974, 2018.

[8] R. Mohan, R. K. Srinivas, and S. Ganapaty, "Hepatoprotective activity of the leaves of Abutilon crispum (Linn) Medikus," World Journal of Pharmacy and Pharmaceutical, vol. 3, pp. 774779, 2015.

[9] I. O. Lima, V. B. Costa, W. N. Matias et al., "Biological activity of Herissantia crispa (L.) Brizicky," Revista Brasileira de Farmacognosia, vol. 19, no. 1b, pp. 249-254, 2009.

[10] R. Mohan and S. Ganapaty, "Anti-Inflammatory and Toxicity Studies of the Leaves of Abutilon crispum," International Journal for Pharmaceutical Research Scholars, vol. 4, pp. 108-111, 2015.

[11] H. G. Kim, W. S. Yang, G.-H. Sung et al., "IKK $\beta$-Targeted AntiInflammatory Activities of a Butanol Fraction of Artificially Cultivated Cordyceps pruinosa Fruit Bodies," Evidence-Based Complementary and Alternative Medicine, vol. 2014, Article ID 562467, 12 pages, 2014.

[12] L. F. Ang, M. F. Yam, Y. T. Fung, P. K. Kiang, and Y. Darwin, "HPLC Method for Simultaneous Quantitative Detection of Quercetin and Curcuminoids in Traditional Chinese Medicines," Journal of Pharmacopuncture, vol. 17, no. 4, pp. 3649, 2014.

[13] M. J. Hossen, W. S. Yang, D. Kim, A. Aravinthan, J.-H. Kim, and J. Y. Cho, "Thymoquinone: an IRAK1 inhibitor with in vivo and in vitro anti-inflammatory activities," Scientific Reports, vol. 7, Article ID 42995, 2017.

[14] Y. Jung, S. E. Byeon, D. S. Yoo et al., "8-(Tosylamino)quinoline inhibits macrophage-mediated inflammation by suppressing NF- $\kappa$ B signaling," Acta Pharmacologica Sinica, vol. 33, no. 8, pp. 1037-1046, 2012. 
[15] K.-S. Baek, Y.-S. Yi, Y.-J. Son et al., "Comparison of anticancer activities of Korean red ginseng-derived fractions," Journal of Ginseng Research, vol. 41, no. 3, pp. 386-391, 2017.

[16] J. G. Park, Y.-J. Son, B. C. Yoo et al., "Syk Plays a Critical Role in the Expression and Activation of IRAK1 in LPS-Treated Macrophages," Mediators of Inflammation, vol. 2017, Article ID 1506248, 9 pages, 2017.

[17] M. J. Hossen, Y. D. Hong, K.-S. Baek et al., "In vitro antioxidative and anti-inflammatory effects of the compound K-rich fraction BIOGF1K, prepared from Panax ginseng," Journal of Ginseng Research, vol. 41, no. 1, pp. 43-51, 2017.

[18] J. G. Park, Y.-J. Son, M.-Y. Kim, and J. Y. Cho, "Syk and IRAK1 contribute to immunopharmacological activities of anthraquinone-2-carboxlic acid," Molecules, vol. 21, no. 6, article no. 809, 2016.

[19] J.-O. Lee, D. Jeong, M.-Y. Kim, and J. Y. Cho, "ATP-Binding Pocket-Targeted Suppression of Src and Syk by Luteolin Contributes to Its Anti-Inflammatory Action," Mediators of Inflammation, vol. 2015, 2015.

[20] S. H. Kim, J. G. Park, J. Lee et al., "The dietary flavonoid Kaempferol mediates anti-inflammatory responses via the Src, Syk, IRAK1, and IRAK4 molecular targets," Mediators of Inflammation, vol. 2015, Article ID 904142, 15 pages, 2015.

[21] S. Y. Han, Y. Yi, S. Jeong et al., "Ethanol Extract of Lilium Bulbs Plays an Anti-Inflammatory Role by Targeting the IKK[Formula: see text]/[Formula: see text]-Mediated NF[Formula: see text]B Pathway in Macrophages," American Journal of Chinese Medicine, vol. 46, no. 06, pp. 1281-1296, 2018.

[22] A. Gomaa, M. Samy, S. Desoukey, and M. Kamel, "Phytochemistry and pharmacological activities of genus Abutilon: a review (1972-2015)," Journal of advanced Biomedical and Pharmaceutical Sciences, vol. 1, no. 2, pp. 56-74, 2018.

[23] J. N. Sharma, A. Al-Omran, and S. S. Parvathy, "Role of nitric oxide in inflammatory diseases," Inflammopharmacology, vol. 15 , no. 6, pp. 252-259, 2007.

[24] M. Taghavi, A. Khosravi, E. Mortaz, D. Nikaein, and S. S. Athari, "Role of pathogen-associated molecular patterns (PAMPS) in immune responses to fungal infections," European Journal of Pharmacology, vol. 808, pp. 8-13, 2017.

[25] C. Alexander and E. T. Rietschel, "Bacterial lipopolysaccharides and innate immunity," Journal of Endotoxin Research, vol. 7, no. 3, pp. 167-202, 2001.

[26] Z. Huang, X. Yi, Y. Chen et al., "Pretreatment of Pam3CSK4 attenuates inflammatory responses caused by systemic infection of methicillin-resistant Staphylococcus aureus in mice," Biomedicine \& Pharmacotherapy, vol. 95, pp. 1684-1692, 2017.

[27] P. J. Barnes, "How corticosteroids control inflammation: quintiles Prize Lecture 2005," British Journal of Pharmacology, vol. 148, no. 3, pp. 245-254, 2006.

[28] V. García-Mediavilla, I. Crespo, P. S. Collado et al., "The anti-inflammatory flavones quercetin and kaempferol cause inhibition of inducible nitric oxide synthase, cyclooxygenase2 and reactive $\mathrm{C}$-protein, and down-regulation of the nuclear factor kappaB pathway in Chang Liver cells," European Journal of Pharmacology, vol. 557, no. 2-3, pp. 221-229, 2007.

[29] M. Mohan, S. Paty, and B. Sudha, "Isolation and characterization of phytosteroid and Triterpenods from leaves of abutilon crispum," International Journal of Pharma and Bio Sciences, vol. 8, no. 3, 2017.

[30] W. S. Yang, D. Jeong, Y.-S. Yi et al., "IRAK1/4-Targeted AntiInflammatory Action of Caffeic Acid," Mediators of Inflammation, vol. 2013, Article ID 518183, 12 pages, 2013.
[31] P.-H. Nguyen, B. T. Zhao, J. H. Lee, Y. H. Kim, B. S. Min, and M. H. Woo, "Isolation of benzoic and cinnamic acid derivatives from the grains of Sorghum bicolor and their inhibition of lipopolysaccharide-induced nitric oxide production in RAW 264.7 cells," Food Chemistry, vol. 168, pp. 512-519, 2015.

[32] Y. Ding, H. T. Nguyen, S. I. Kim, H. W. Kim, and Y. H. Kim, "The regulation of inflammatory cytokine secretion in macrophage cell line by the chemical constituents of Rhus sylvestris," Bioorganic \& Medicinal Chemistry Letters, vol. 19, no. 13, pp. 3607-3610, 2009.

[33] E. McNeill, M. J. Crabtree, N. Sahgal et al., "Regulation of iNOS function and cellular redox state by macrophage Gch1 reveals specific requirements for tetrahydrobiopterin in NRF2 activation," Free Radical Biology \& Medicine, vol. 79, pp. 206216, 2015.

[34] R. Zamora, Y. Vodovotz, and T. R. Billiar, "Inducible nitric oxide synthase and inflammatory diseases," Molecular Medicine, vol. 6, no. 5, pp. 347-373, 2000.

[35] V. Piras and K. Selvarajoo, "Beyond MyD88 and TRIF pathways in Toll-like receptor signaling," Frontiers in Immunology, vol. 5, article 70, 2014. 


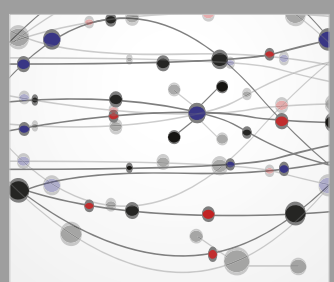

The Scientific World Journal
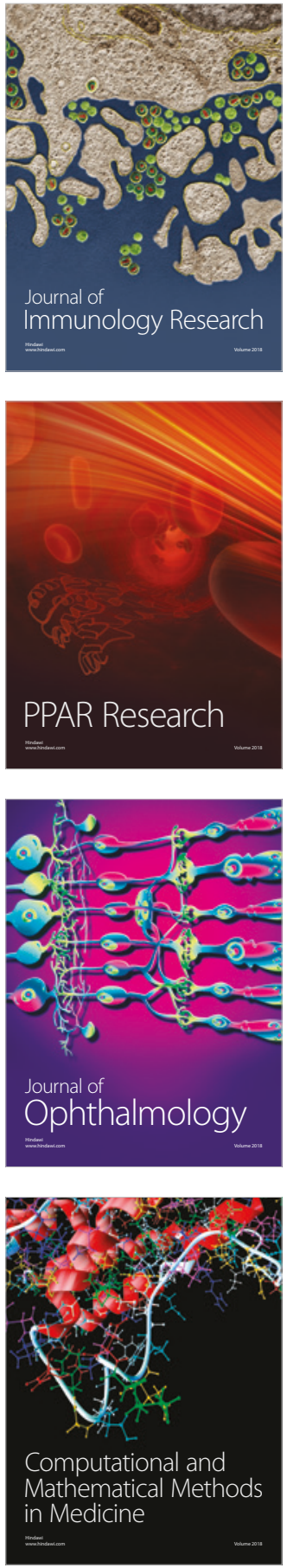

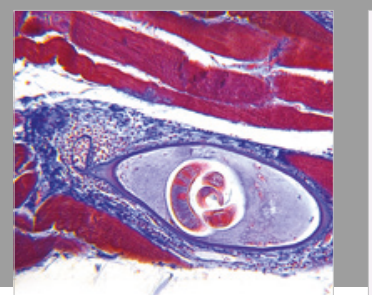

Gastroenterology Research and Practice

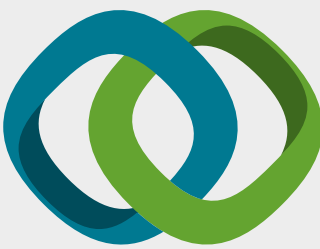

\section{Hindawi}

Submit your manuscripts at

www.hindawi.com
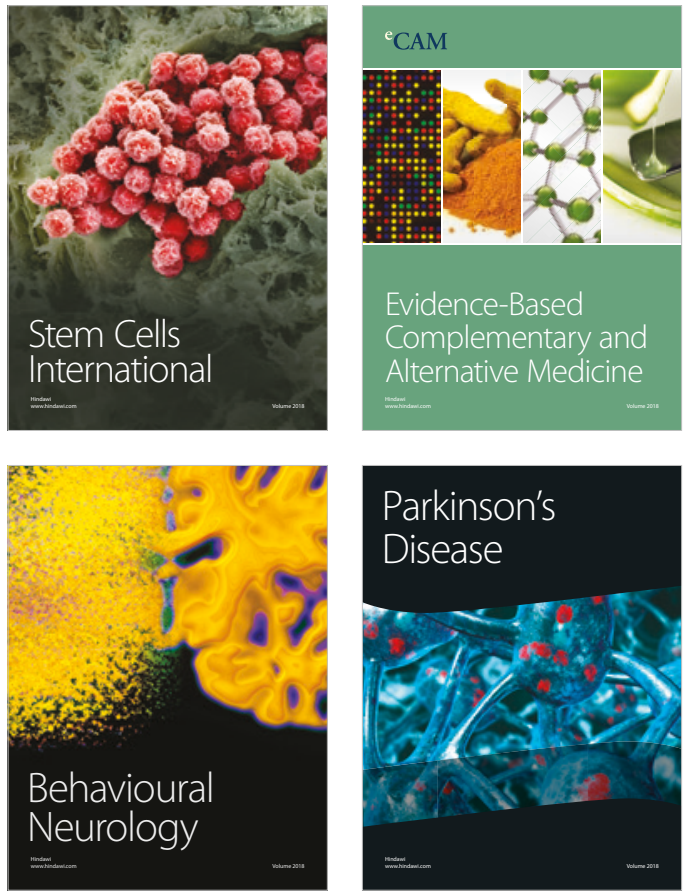

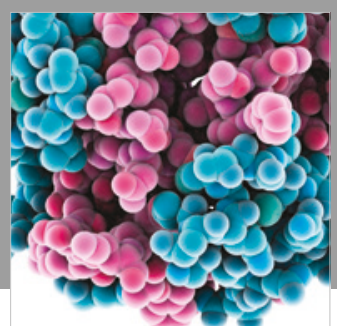

ournal of

Diabetes Research

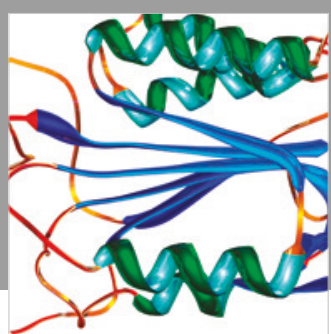

Disease Markers
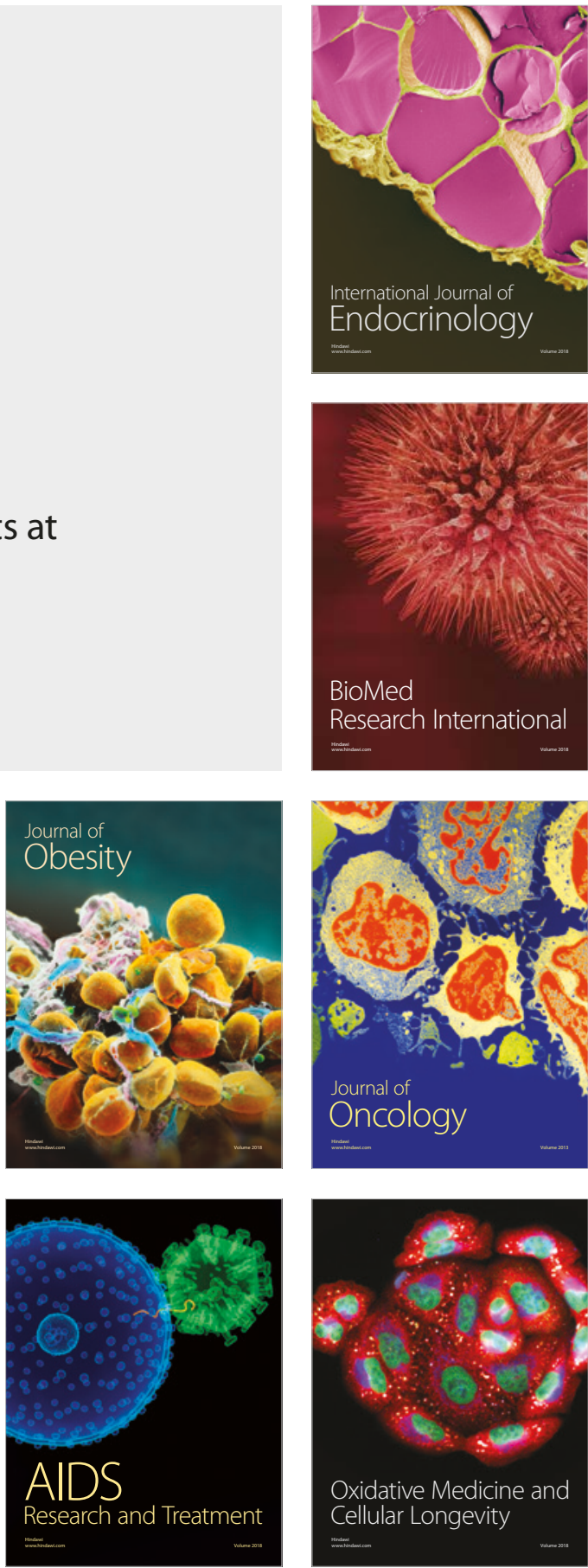